\title{
The 3D-Computational Study of the Heat Transfer Enhancement by Using of the Longitudinal and Transversal Internal Fins in a Heated Horizontal Pipe
}

\author{
Sofiane Touahri and Toufik Boufendi
}

\begin{abstract}
In this work, we numerically study the three-dimensional mixed convection heat transfer in a horizontal pipe equipped by longitudinal and transversal attached fins on its internal surface for the solar application such as the water or air heating with solar panel. The pipe and fins are heated by an electrical intensity passing through their small thickness. The longitudinal fins studied number is: 2 vertical, 4 and 8 fins, while the number of transversal fins is 8 fins. The considered heights fin are $H=0.12 \mathrm{~cm}$ and $H=0.18 \mathrm{~cm}$. The convection in the fluid domain is conjugated to thermal conduction in the pipe and fins solid thickness. The physical properties of the fluid are thermal dependant. The heat losses from the external pipe surface to the surrounding ambient are considered. The model equations of continuity, momenta and energy are numerically solved by a finite volume method with a second order spatiotemporal discretization. As expected for the longitudinal fins, the axial Nusselt number increase with increasing of number and height of fins. For $\mathrm{Gr}=5.110^{5}$, the average Nusselt number without fins is equal to 13.144. The introduce of longitudinal fins gives an average Nusselt number equal to $16.83,22.523$ and 32.066 for 2 vertical, 4 and 8 fins respectively. The participation of fins located in the lower part of the tube on the improvement of heat transfer is higher than the participation of the upper fins. On the other hand, the longitudinal fins participate directly on increase of the heat transfer; this is justified by the large local Nusselt number along the interface of the fins. This participation is moderate in the case of transverse fins, these latter are used to mix the fluid for increased the local Nusselt number to the interface in the cylindrical axial sections following the transversal fins.
\end{abstract}

Index Terms - Conjugate heat transfer, internal fins, mixed convection, numerical simulation.

\section{INTRODUCTION}

Finned tubes are often used in many engineering sectors for extend the contact surface between the tube wall and the fluid and improve the heat transfer; the researchers have studied the problem of optimizing the shape and geometry of attached fins in order to increase heat transfer effectiveness. Most of the studies performed on this optimization consider longitudinal fins which have symmetrical lateral profiles; this assumption simplifies the treatment of the problem with regard to the boundary conditions and gives symmetrical results concerning velocity and temperature profiles. Many investigations, both experimental and numerical, have been conducted for different kinds of internally finned tubes. Reference [1], presents an analytical model for fully

Manuscript received June 10, 2015; revised October 19, 2015

The authors are with Energy Physics Laboratory, Physics Department, Mentouri Brothers University, Constantine, Algeria (e-mail: sofianetouahri04@yahoo.fr, boufendit@yahoo.fr). developed turbulent air flow in internally finned tubes and annuli. In their study, the longitudinal attached fins in the inner wall were considered. The thermal boundary conditions were constant heat flux at the inner surface, the results of this study consisted of heat transfer and pressure drop coefficients. A combined numerical and experimental study of plate-fin and tube heat exchangers was examined by [2]. In their study, the detailed numerical results of pressure drop and heat transfer coefficient are presented. Reference [3] conducts a numerical study on hydrodynamically fully developed, thermally developing flow inside circular tubes with internal longitudinal fins having tapered lateral profiles. The results showed significant heat transfer enhancement with the inclusion of internal fins. Water and engine oil were assumed as fluids in their numerical studies, and they concluded water to be a better coolant as compared to engine oil. In [4], a numerical study of thermally developing flow in an elliptical duct with four longitudinal internal fins of zero thickness is considered. A control volume based on finite difference technique was used in the numerical analysis and an optimum value of the local Nusselt number was obtained as a function of the fin height. Reference [5] performs an experimental analysis of heat transfer in an internally finned tube, the experimental results were compared with results from the smooth channel tube, and a significant improvement in heat transfer was observed for internally finned cases. Similar studies were also examined numerically and experimentally by [6]-[10].

In this work, we studied numerically the heat transfer by mixed convection in horizontal pipe equipped by longitudinal and transversal attached fins on its internal wall. The mixed convection is conjugate with thermal conduction in the pipe and fins walls. The physical properties of the fluid are thermo- dependent and the heat losses with the external environment are considered. The objective of our work is study the enhancement gives to the heat transfer by using different fins shape.

\section{The Geometry And Mathematical Model}

Fig. 1 illustrates the problem geometry. We consider a long horizontal pipe having a length $L=1 \mathrm{~m}$, an inside diameter $D_{i}=0.96 \mathrm{~cm}$ and an external diameter $D_{o}=1 \mathrm{~cm}$, this later is equipped by longitudinal and transversal attached fins on its internal surface. The longitudinal fins are fixed at $(\theta=0)$, $(\theta=\pi / 4),(\theta=\pi / 2),(\theta=3 \pi / 4),(\theta=\pi),(\theta=5 \pi / 4),(\theta=3 \pi / 2)$ and $(\theta=7 \pi / 4)$ while the transversal fins are attached at $z^{*}=9.4404$, $z^{*}=19.8574, \quad z^{*}=30.2744, \quad z^{*}=40.6914, \quad z^{*}=51.1084$, $z^{*}=61.5254, z^{*}=75.8488$ and $z^{*}=82.3594$. The pipe and fins is 
made of Inconel having a thermal conductivity $K_{s}=20 \mathrm{~W} / \mathrm{m}$ $\mathrm{K}$. An electric current passing along the pipe (in the solid thickness) produced a heat generation by the Joule effect. This heat is transferred to distilled water flow in the pipe. At the entrance the flow is of Poiseuille type with an average axial velocity equal to $7.210^{-2} \mathrm{~m} / \mathrm{s}$ and a constant temperature of $15^{\circ} \mathrm{C}$. The density is a linear function of temperature and the Boussinesq approximation is applied. The physical principles involved in this problem are well modeled by the following non dimensional conservation partial differential equations with their initial and boundary conditions.

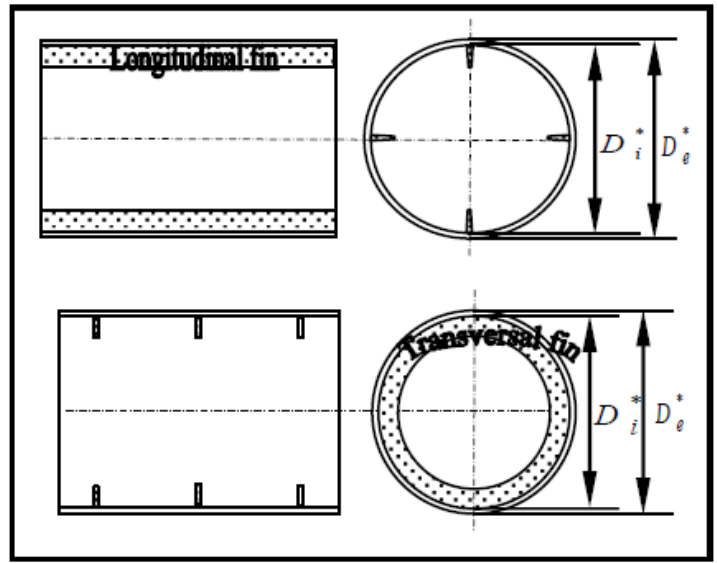

Fig. 1. Pipe with longitudinal fin (a) ; transversal fin (b).

A. Modeling Equations

$$
\text { At } t^{*}=0: \quad V_{r}^{*}=V_{\theta}^{*}=V_{z}^{*}=T^{*}=0
$$

At $t^{*}>0$ :

Mass conservation equation

$$
\frac{1}{r^{*}} \frac{\partial}{\partial r^{*}}\left(r^{*} V_{r}^{*}\right)+\frac{1}{r^{*}} \frac{\partial V_{\theta}^{*}}{\partial \theta}+\frac{\partial V_{z}^{*}}{\partial z^{*}}=0
$$

Radial momentum conservation equation

$$
\begin{aligned}
& \frac{\partial V_{r}^{*}}{\partial t^{*}}+\frac{1}{r^{*}} \frac{\partial}{\partial r^{*}}\left(r^{*} V_{r}^{*} V_{r}^{*}\right)+\frac{1}{r^{*}} \frac{\partial}{\partial \theta}\left(V_{\theta}^{*} V_{r}^{*}\right)+ \\
& \frac{\partial}{\partial z^{*}}\left(V_{z}^{*} V_{r}^{*}\right)-\frac{V_{\theta}^{* 2}}{r^{*}}=-\frac{\partial P^{*}}{\partial r^{*}}+\frac{G r_{0}^{*}}{\operatorname{Re}_{0}^{2}}(\cos \theta) T^{*}+ \\
& \frac{1}{\operatorname{Re}_{0}}\left[\frac{1}{r^{*}} \frac{\partial}{\partial r^{*}}\left(r^{*} \tau_{r r}^{*}\right)+\frac{1}{r^{*}} \frac{\partial}{\partial \theta}\left(\tau_{r \theta}^{*}\right)-\frac{\tau_{\theta \theta}^{*}}{r^{*}}+\frac{\partial}{\partial z^{*}}\left(\tau_{r z}^{*}\right)\right]
\end{aligned}
$$

Angular momentum conservation equation

$$
\begin{aligned}
& \frac{\partial V_{\theta}^{*}}{\partial t^{*}}+\frac{1}{r^{*}} \frac{\partial}{\partial r^{*}}\left(r^{*} V_{r}^{*} V_{\theta}^{*}\right)+\frac{1}{r^{*}} \frac{\partial}{\partial \theta}\left(V_{\theta}^{*} V_{\theta}^{*}\right)+ \\
& \frac{\partial}{\partial z^{*}}\left(V_{z}^{*} V_{\theta}^{*}\right)+\frac{V_{r}^{*} V_{\theta}^{*}}{r^{*}}=-\frac{1}{r^{*}} \frac{\partial P^{*}}{\partial \theta}-\frac{G r_{0}^{*}}{\operatorname{Re}_{0}^{2}} \sin \theta T^{*}+ \\
& \frac{1}{\operatorname{Re}_{0}}\left[\frac{1}{r^{* 2}} \frac{\partial}{\partial r^{*}}\left(r^{* 2} \tau_{\theta r}^{*}\right)+\frac{1}{r^{*}} \frac{\partial}{\partial \theta}\left(\tau_{\theta \theta}^{*}\right)+\frac{\partial}{\partial z^{*}}\left(\tau_{\theta z}^{*}\right)\right]
\end{aligned}
$$

Axial momentum conservation equation:

$$
\begin{aligned}
& \frac{\partial \mathrm{V}_{\mathrm{z}}^{*}}{\partial \mathrm{t}^{*}}+\frac{1}{\mathrm{r}^{*}} \frac{\partial}{\partial \mathrm{r}^{*}}\left(\mathrm{r}^{*} \mathrm{~V}_{\mathrm{r}}^{*} \mathrm{~V}_{\mathrm{z}}^{*}\right)+\frac{1}{\mathrm{r}^{*}} \frac{\partial}{\partial \theta}\left(\mathrm{V}_{\theta}^{*} \mathrm{~V}_{\mathrm{z}}^{*}\right)+ \\
& \frac{\partial}{\partial \mathrm{z}^{*}}\left(\mathrm{~V}_{\mathrm{z}}^{*} \mathrm{~V}_{\mathrm{z}}^{*}\right)=-\frac{\partial \mathrm{P}^{*}}{\partial \mathrm{z}^{*}}+ \\
& \frac{1}{\operatorname{Re}_{0}}\left[\frac{1}{\mathrm{r}^{*}} \frac{\partial}{\partial \mathrm{r}^{*}}\left(\mathrm{r}^{*} \tau_{\mathrm{rz}}^{*}\right)+\frac{1}{\mathrm{r}^{*}} \frac{\partial}{\partial \theta}\left(\tau_{\theta \mathrm{z}}^{*}\right)+\frac{\partial}{\partial \mathrm{z}^{*}}\left(\tau_{\mathrm{zz}}^{*}\right)\right]
\end{aligned}
$$

Energy Conservation Equation

$$
\begin{aligned}
& \frac{\partial T^{*}}{\partial t^{*}}+\frac{1}{r^{*}} \frac{\partial}{\partial r^{*}}\left(r^{*} V_{r}^{*} T^{*}\right)+\frac{1}{r^{*}} \frac{\partial}{\partial \theta}\left(V_{\theta}^{*} T^{*}\right)+ \\
& \frac{\partial}{\partial z^{*}}\left(V_{z}^{*} T^{*}\right)=G^{*}- \\
& \frac{1}{\operatorname{Re}_{0} \operatorname{Pr}_{0}}\left[\frac{1}{r^{*}} \frac{\partial}{\partial r^{*}}\left(r^{*} q_{r}^{*}\right)+\frac{1}{r^{*}} \frac{\partial}{\partial \theta}\left(q_{\theta}^{*}\right)+\frac{\partial}{\partial z^{*}}\left(q_{z}^{*}\right)\right]
\end{aligned}
$$

where $G^{*}=\left\{\begin{array}{l}K_{S}^{*} /\left(\operatorname{Re}_{0} \operatorname{Pr}_{0}\right) \text { in the solid } \\ 0 \quad \text { in the fluid }\end{array}\right.$

The viscous stress tensor components are:

$$
\begin{array}{ll}
\tau_{r r}^{*}=2 \mu^{*} \frac{\partial V_{r}^{*}}{\partial r^{*}}, & \tau_{r \theta}^{*}=\tau_{\theta r}^{*}=\mu^{*}\left\lfloor r^{*} \frac{\partial}{\partial r^{*}}\left(\frac{V_{\theta}^{*}}{r^{*}}\right)+\frac{1}{r^{*}} \frac{\partial V_{r}^{*}}{\partial \theta}\right\rfloor \\
\left.\tau_{\theta \theta}^{*}=2 \mu^{*} \mid \frac{1}{r^{*}} \frac{\partial V_{\theta}^{*}}{\partial \theta}+\frac{V_{r}^{*}}{r^{*}}\right\rfloor, & \tau_{\theta z}^{*}=\tau_{z \theta}^{*}=\mu^{*}\left\lfloor\frac{\partial V_{\theta}^{*}}{\partial z^{*}}+\frac{1}{r^{*}} \frac{\partial V_{z}^{*}}{\partial \theta}\right\rfloor \\
\tau_{z}^{*}=2 \mu^{*} \frac{\partial V_{z}^{*}}{\partial z^{*}}, & \tau_{z r}^{*}=\tau_{r z}^{*}=\mu^{*}\left\lfloor\frac{\partial V_{z}^{*}}{\partial r^{*}}+\frac{1}{r^{*}} \frac{\partial V_{r}^{*}}{\partial z^{*}}\right\rfloor
\end{array}
$$

The heat fluxes are:

$$
q_{r}^{*}=-K^{*} \frac{\partial T^{*}}{\partial r^{*}} \quad, \quad q_{\theta}^{*}=-\frac{K^{*}}{r^{*}} \frac{\partial T^{*}}{\partial \theta^{*}} \quad \text { and } \quad q_{Z}^{*}=-K^{*} \frac{\partial T^{*}}{\partial z^{*}}
$$

\section{B. The Boundary Conditions}

The previous differential equations are solved with the following boundary conditions:

At the pipe entrance : $\mathrm{z}^{*}=0$

In the fluid domain : $0 \leqslant r^{*} \leqslant$.5 and $0 \leqslant \theta \leqslant 2 \pi$ :

$$
V_{r}^{*}=V_{\theta}^{*}=T^{*}=0, \quad V_{z}^{*}=2\left(1-4 r^{* 2}\right)
$$

In the solid domain : $0.5 \leqslant r^{*} \leqslant 0.5208$ and $0 \leqslant \theta \leqslant 2 \pi$ :

$$
V_{r}^{*}=V_{\theta}^{*}=V_{z}^{*}=T^{*}=0
$$

At the pipe exit : $z^{*}=104.17$

In the fluid domain: $0 \leqslant r^{*} \leqslant .5$ and $0 \leqslant \theta \leqslant 2 \pi$ :

$$
\frac{\partial V_{r}^{*}}{\partial z^{*}}=\frac{\partial V_{\theta}^{*}}{\partial z^{*}}=\frac{\partial V_{z}^{*}}{\partial z^{*}}=\frac{\partial}{\partial z^{*}}\left(K^{*} \frac{\partial T^{*}}{\partial z^{*}}\right)=0
$$

In the solid domain: $0.5 \leqslant r^{*} \leqslant 0.5208$ and $0 \leqslant \theta \leqslant 2 \pi$ :

$$
V_{r}^{*}=V_{\theta}^{*}=V_{z}^{*}=\frac{\partial}{\partial z^{*}}\left(K^{*} \frac{\partial T^{*}}{\partial z^{*}}\right)=0
$$

At the outer wall of the pipe : $r^{*}=0.5208$

$$
\begin{aligned}
& \left\{\begin{array}{l}
V_{r}^{*}=V_{\theta}^{*}=V_{z}^{*}=0 \\
-K^{*} \frac{\partial T^{*}}{\partial r^{*}}=\frac{\left(h_{r}+h_{c}\right) D_{i}}{K_{0}} T^{*}
\end{array}\right. \\
& h_{r}=\varepsilon \sigma\left(T^{2}+T_{\infty}^{2}\right)\left(T+T_{\infty}\right)
\end{aligned}
$$

The emissivity of the outer wall $\varepsilon$ is arbitrarily chosen to 0.9 while $h_{c}$ is derived from the correlation of [11] valid for all Pr and for Rayleigh numbers in the range $10^{-6} \leq R a \leq 10^{9}$. 


$$
\begin{aligned}
N u & =\left[h_{c} D_{i} / K_{\text {air }}\right] \\
& =\left[0.6+\left(0.387 R a^{1 / 6} /\left(1+\left(0.559 / P r_{\text {air }}\right)^{9 / 16}\right)^{8 / 27}\right)\right]^{2} \\
\text { with } \quad & R a=\frac{g \beta\left[T\left(R_{o}, \theta, z\right)-T_{\infty}\right] D_{o}^{3}}{\alpha_{\text {air }} v_{\text {air }}}, \quad P r_{\text {air }}=v_{\text {air }} / \alpha_{\text {air }}
\end{aligned}
$$

In this expressions of the Rayleigh and the Prandtl numbers the thermophysical properties of the air ambient are evaluated at the local film temperature given as: $T_{\text {film }}=\left[T\left(R_{o}, \theta, z\right)+T_{\infty}\right] / 2$.

In our calculations we have considered the solid as a fluid with a dynamic viscosity equal to $10^{30}$. This very large viscosity within the solid domain ensures that the velocity of this part remains null and consequently the heat transfer is only by conduction deducted from (6).

\section{The Nusselt Numbers}

At the cylindrical solid-fluid interface, the local Nusselt number is defined as:

$$
N u\left(\theta, z^{*}\right)=\frac{h\left(\theta, z^{*}\right) D_{i}}{K_{0}}=\left[\frac{\left.\left(K^{*} \partial T^{*} / \partial r^{*}\right)\right|_{r^{*}=0.5}}{T^{*}\left(0.5, \theta, z^{*}\right)-T_{b}^{*}\left(z^{*}\right)}\right]
$$

The axial Nusselt number for the cylindrical interface is:

$$
N u\left(z^{*}\right)=\frac{1}{2 \pi} \int_{0}^{2 \pi} N u\left(\theta, z^{*}\right) d \theta
$$

Finally, we can define an average Nusselt number for the whole cylindrical solid-fluid interface:

$$
N u_{A}=\frac{1}{(2 \pi)(104.17)} \int_{0}^{2 \pi} \int_{0}^{104.17} N u\left(\theta, z^{*}\right) d z^{*} d \theta
$$

At the longitudinal fin interface, the local Nusselt number is defined as:

$$
N u\left(r^{*}, z^{*}\right)=\frac{h\left(r^{*}, z^{*}\right) D_{h}}{K_{0}}=\frac{\left.\left(K^{*} / r^{*}\right)\left(\partial T^{*} / \partial \theta\right)\right|_{\theta=\theta_{\text {fin }}}}{T^{*}\left(r^{*}, \theta_{f i n}, z^{*}\right) T_{m}^{*}\left(z^{*}\right)}
$$

The axial Nusselt number for the longitudinal fin interface is:

$$
N u\left(z^{*}\right)=\frac{1}{H^{*}}{ }_{R_{i}^{*}}^{R_{i}^{*}} \int_{H^{*}}^{*} N u\left(r^{*}, z^{*}\right) d r^{*}=\frac{1}{H^{*}}{ }_{R_{i}^{*}}^{R_{i}^{*}} \int_{H^{*}} \frac{\left.\left(K^{*} / r^{*}\right)\left(\partial T^{*} / \partial \theta\right)\right|_{\theta=\theta_{m}}}{T^{*}\left(r^{*}, \theta_{f i n}, z^{*}\right) T_{m}^{*}\left(z^{*}\right)} d r^{*}
$$

The average Nusselt number for the longitudinal fin interface is defined as:

$$
N u_{A}=\frac{1}{L^{*}} \int_{0}^{L^{*}} N u\left(z^{*}\right) d z^{*}
$$

At the transversal fin interface, the local Nusselt number is

$$
N u\left(r^{*}, \theta\right)=\frac{h\left(r^{*}, \theta\right) D_{h}}{K_{0}}=\frac{\left.\left(K^{*}\right)\left(\partial T^{*} / \partial z\right)\right|_{z=z_{\text {im }}}}{T^{*}\left(r^{*}, \theta, z_{\text {fin }}^{*}\right) T_{m}^{*}\left(z^{*}\right)}
$$

The axial Nusselt number for the transversal fin interface is defined as:

$$
N u\left(z^{*}\right)=\frac{1}{(2 \pi) H^{*}} \int_{0}^{2 \pi} \int_{R_{i}^{*}}^{R_{H_{i}^{*}}^{*}} \frac{\left.\left(K^{*}\right)\left(\partial T^{*} / \partial z\right)\right|_{z^{*}=z_{\text {fi }}^{*}}}{T^{*}\left(r^{*}, \theta, z_{\text {fin }}^{*}\right) T_{m}^{*}\left(z^{*}\right)} d r^{*} d \theta
$$

\section{NumericAl RESOLUTION}

For the numerical solution of modeling equations, we used the finite volume method well described by [12]; the using of this method involves the discretization of the physical domain into a discrete domain constituted of finite volumes where the modeling equations are discretized in a typical volume. We used a temporal discretization with a truncation error of $\left(\Delta t^{*}\right)^{2}$ order. The convective and nonlinear terms have been discretized according to the Adams-Bashforth numerical scheme, with a truncation error of $\left(\Delta t^{*}\right)^{2}$ order, the diffusive and pressure terms are implicit. Regarding the spatial discretization, we used the central differences pattern with a truncation error of $\left(\Delta r^{*}\right)^{2},\left(\Delta \theta^{*}\right)^{2}$ and $\left(\Delta z^{*}\right)^{2}$ order. So our spatio-temporal discretization is second order. The mesh used contains $52 \times 88 \times 162$ points in the radial, angular and axial directions. The considered time step is $\Delta t^{*}=5.10^{4}$ and the time marching is pursued until the steady state is reached. The steady state is controlled by the satisfaction of the global mass and energy balances as well as the leveling off of the time evolution of the hydrodynamic and thermal fields.

The accuracy of the results of our numerical code has been tested by the comparison of our results with those of other researchers. A comparison with the results of [13] who studied the non conjugate and the conjugate mixed convection heat transfer in a pipe with constant physical properties of the fluid. Some of their results concern the simultaneously developing heat transfer and fluid flow in a uniformly heated inclined pipe $\left(\alpha=40^{\circ}\right)$.The controlling parameters of the problem are: $\operatorname{Re}=500, P r=7.0, G r=10^{4}$ and $10^{6}, L / D_{i}=90, R_{o} / D_{i}=0.583, K_{s} / K_{0}=70$. The used grid is $40 \times 36 \times 182$ in the $\mathrm{r}^{*}, \theta$ and $\mathrm{z}^{*}$ directions, respectively. We reproduced the results of the cited reference with the first order calculus code concerning the conjugate and non conjugate mixed convection. In Fig. 2 we illustrate the axial evolution of the circumferentially averaged Nusselt number. It is seen that there is a good agreement between our results and theirs.

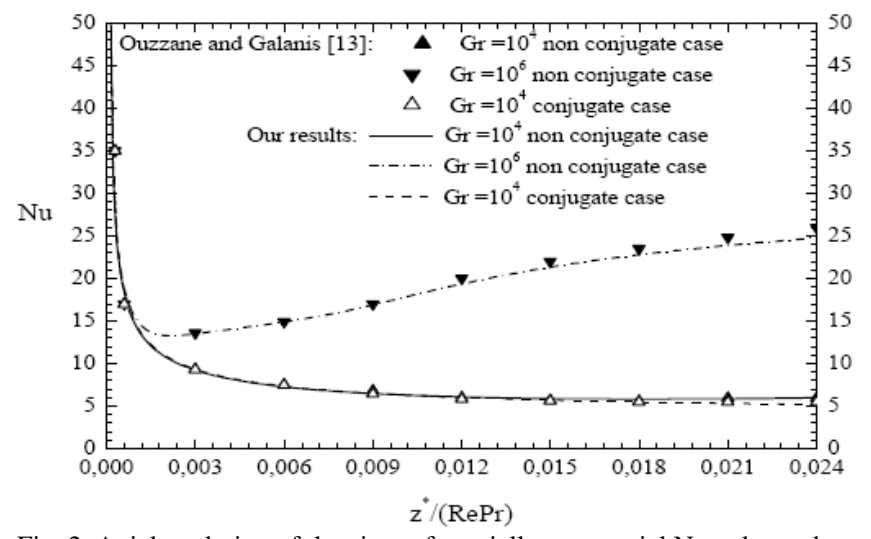

Fig. 2. Axial evolution of the circumferentially mean axial Nusselt number; A comparison with the results of [13].

\section{RESUltS AND Discussion}

\section{A. Development of the Secondary Flow}

All the results presented in this paper were calculated for 
Reynolds number, $\operatorname{Re}=606.85$, and the Prandtl number, $\operatorname{Pr}=8.082$, while the Grashof number is equal to $5.110^{5}$. The obtained flow for the studied cases is characterized by a main flow along the axial direction and a secondary flow influenced by the density variation with temperature, which occurs in the plane $\left(r^{*}-\theta\right)$, this flows are presented for 8 fins in the case of longitudinal fins and 4 fins in the case of transversal fins. In the reference case (forced convection) the transverse motion is nonexistent; the only flow is in axial direction. In the presence of volumetric heating in the pipe and fins wall, a transverse flow exists and explained as follows: the hot fluid moves along the hot wall from the bottom of the outer tube $(\theta=\pi)$ upwards $(\theta=0)$ and moves down from the top to the bottom along the centre of tube. The vertical plane passing through the angles $(\theta=0)$ and $(\theta=\pi)$ is a plane of symmetry. The transverse flow in the $\left(\overline{r^{*}}, \theta\right)$ plane is represented by counter rotating cells; the cells number is proportional to longitudinal fins number. In Fig. 3, we present the secondary flow vectors at the pipe exit for the case of longitudinal fins. For the case of transversal fins, the position of fins is only in selected axial sections. Far from these sections, the secondary motion is similar to that of simple cylindrical pipe. The Fig. 4, illustrates the secondary flow vectors at the fourth transversal fin section $\left(z^{*}=40.6914\right)$.

\section{B. The Axial Flow Development}

At the entrance, the axial flow is axisymmetric with the maximum velocity in the center of the pipe. In the presence of volumetric heating in the pipe and the fins walls, the configuration of the axial flow completely changes because the secondary flow causes an angular variation which has a direct influence on the distribution of axisymmetric axial flow. In Fig. 5, the axial flow is presented at the exit of the pipe for the case of longitudinal fins (height $H^{*}=0.1875$ ). In Fig. 6, the axial flow is presented at the fourth transversal fin section $\left(\mathrm{z}^{*}=40.6914\right)$. Through these figures, it is clear that the axial velocity is zero in the fins walls.

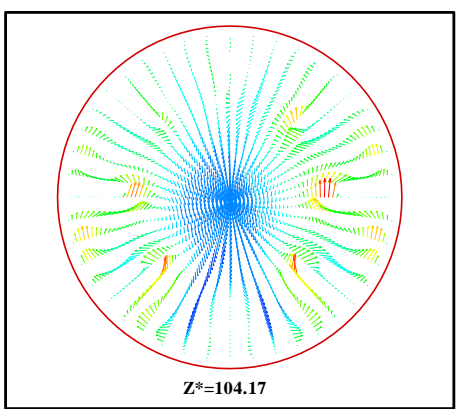

Fig. 3. The secondary flow vectors at the pipe exit for longitudinal fins.

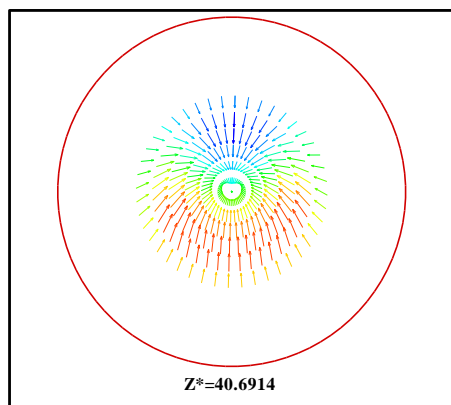

Fig. 4. The secondary flow vectors at the fourth transversal fin section.

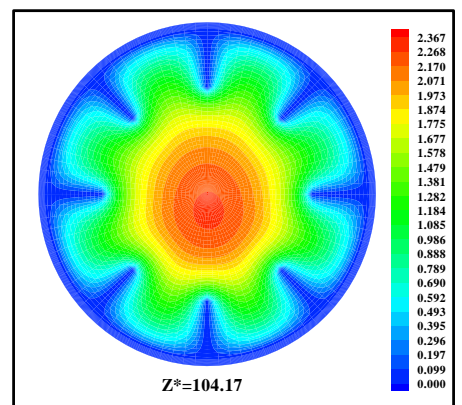

Fig. 5. Axial velocity profiles at the pipe exit for longitudinal fins.

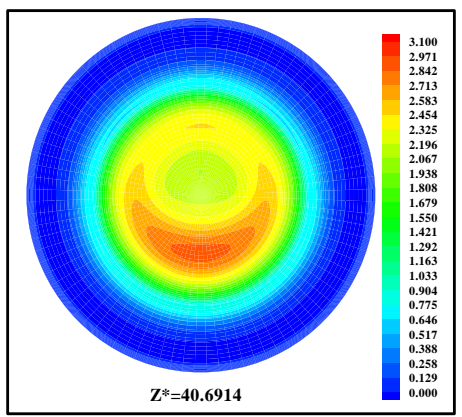

Fig. 6. Axial velocity profiles at the fourth transversal fin section/

\section{Development of the Temperature Field}

In the presence of volumetric heating, a transverse flow exists and thus changes the axisymmetric distribution of fluid and gives it an angular variation, this variation explained as follows: the hot fluid near from the pipe and fins walls moves upwards under the buoyancy force effect, the relatively cold fluid descends down in de centre of the pipe. A permanent generation of heat in the pipe and the fins walls imposes a continuous increase of the temperature of the fluid up to the exit of the pipe. The obtained results show that at given section, the maximum fluid temperature $T^{*}$ is all the time located at $r^{*}=0.5$ and $\theta=0$ (top of solid-fluid interface), because the hot fluid is driven by the secondary motion towards the top of the pipe. The isotherms are presented at the exit of the pipe for the case of longitudinal fins having height $\mathrm{H}^{*}$ equal to 0.1875 in Fig. 7 and at the fourth transversal fin section $\left(z^{*}=40.6914\right)$ in Fig. 8.

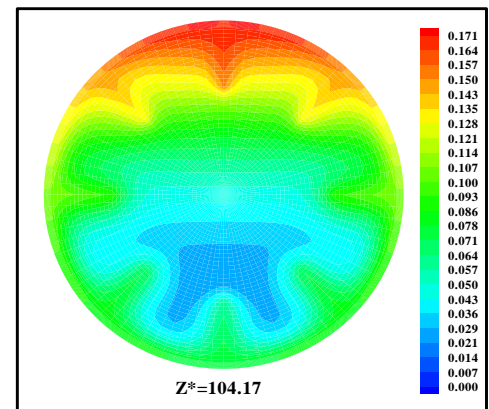

Fig. 7. The isotherms at the pipe exit for longitudinal fins.

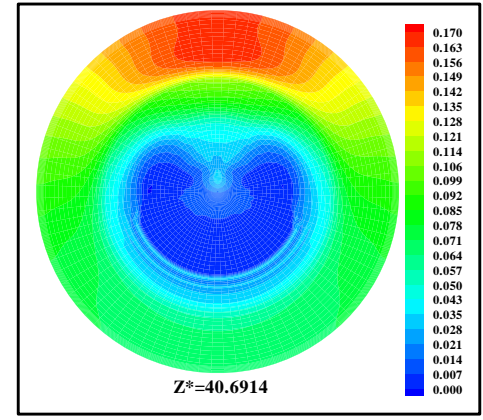

Fig. 8. The isotherms at the fourth transversal fin section. 


\section{The Nusselt Numbers}

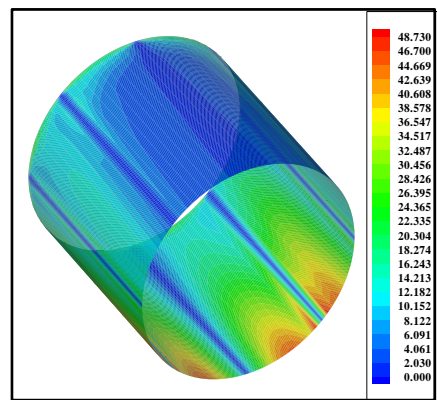

Fig. 9. The local Nusselt number variation at cylindrical interface for longitudinal fins case.

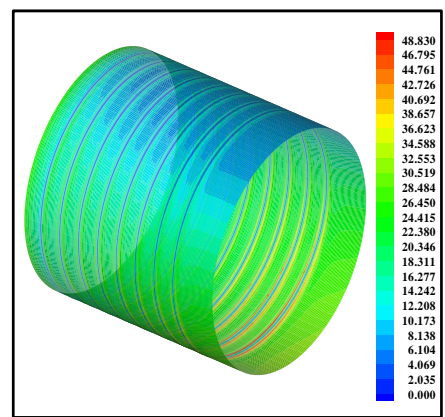

Fig. 10. The local Nusselt number variation at cylindrical interface for transversal fins case.

The phenomenon of heat transfer has been characterized in terms of Nusselt numbers calculated at the inner wall of the pipe, obtained by (17) and those calculated at the fins wall, obtained by (20) and (23). The variation of local Nusselt number at cylindrical interface is presented in Fig. 9 for the case of longitudinal fins and in Fig. 10 for the case of transversal fins. The local Nusselt number of longitudinal fins placed in the right side of the pipe at $(\theta=0, \pi / 4, \pi / 2,3 \pi / 4$, $\pi)$ is shown in Fig. 11. The local Nusselt number takes a maximum value equal to 63.56 on the fin placed at $(\theta=3 \pi / 4)$, $z^{*}=104.17$ and $r^{*}=0.3841$. The comparison of axial Nusselt numbers between finned tube and smooth tube is shown in Fig. 12. Quantitatively, there is a large increase in the axial Nusselt when the number of fins is increased. At the exit of the pipe, the axial Nusselt number is equal to $15.79,23.47$, 31.34 and 47.31 for the cases: smooth tube, tow vertical fins, four fins and eight fins respectively. The average Nusselt numbers for these cases are 13.144, 16.830, 22.523 and 32.066 .

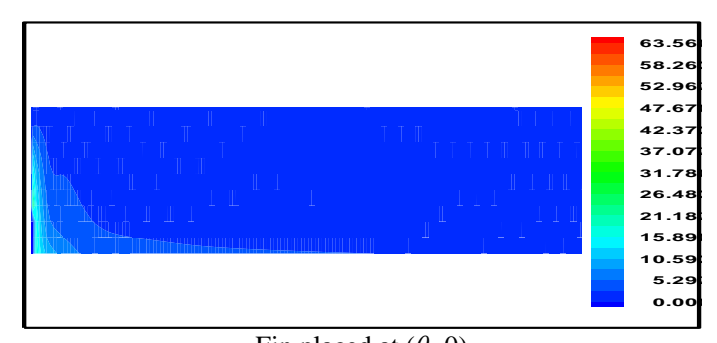

Fin placed at $(\theta=0)$

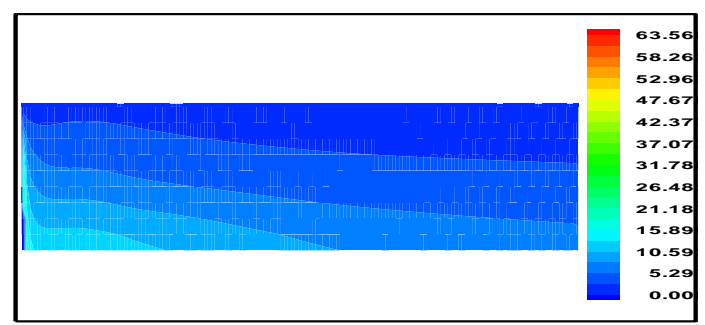

Fin placed at $(\theta=\pi / 4)$
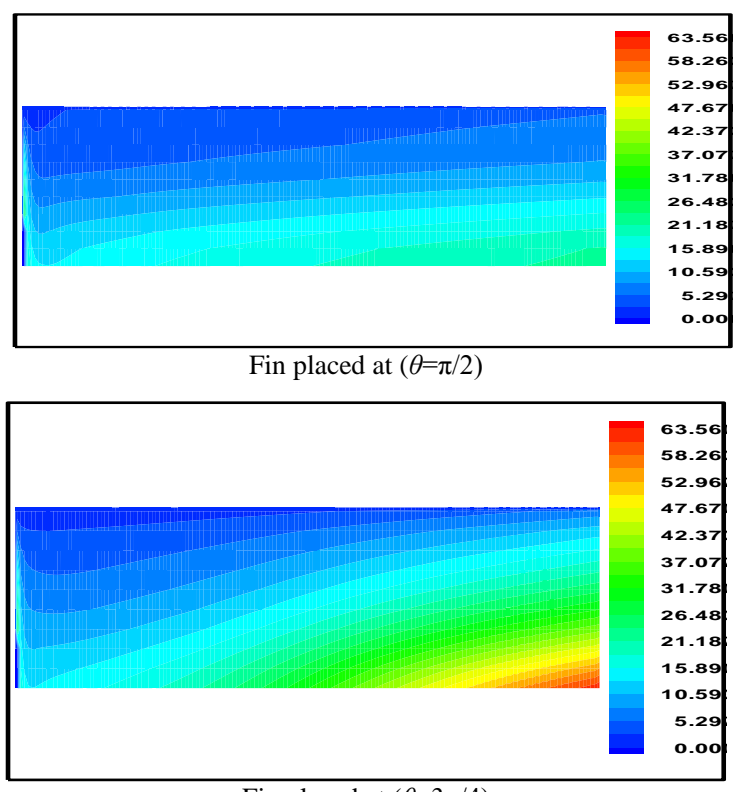

Fin placed at $(\theta=3 \pi / 4)$

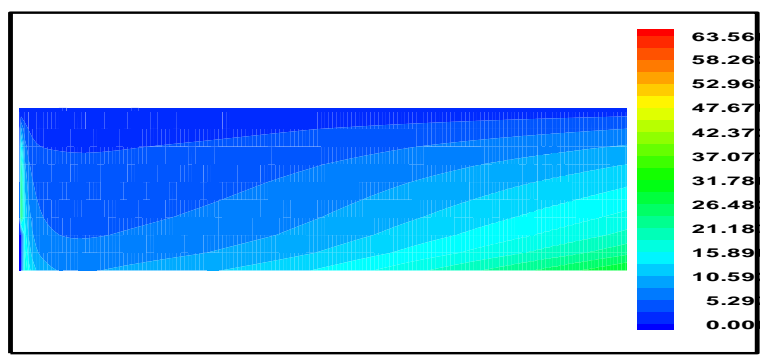

Fin placed at $(\theta=\pi)$

Fig. 11. The local Nusselt number variation at longitudinal fins interface.

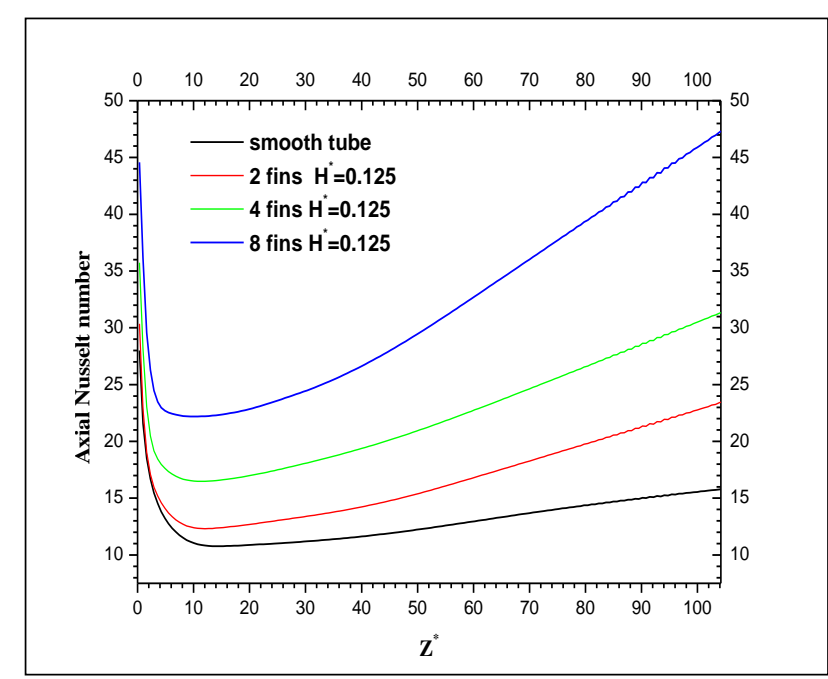

Fig. 12. Variation of axial Nusselt number for the different longitudinal fins studied cases $\left(H^{*}=0.125\right)$.

\section{CONCLUSION}

This study considers the numerical simulation of the three dimensional mixed convection heat transfers in a horizontal pipe equipped by longitudinal and transversal fins attached on its internal surface. The pipe and fins are heated by an electrical intensity passing through its small thickness. The obtained results show that the longitudinal fins participate directly in improving the heat transfer; this is justified by the high local Nusselt number at the interface of longitudinal fins. By against, the transverse fins participate in an indirect way 
in improving the heat transfer their location facing the flow allowed to rearrange the structure of the fluid for each passage through the fins, which is used to mix the fluid and to increase the heat transfer to the cylindrical interfaces. The number and fins height are also important factors in improving the heat transfer.

\section{REFERENCES}

[1] S. V. Patankar, "Analysis of nnuturbulent flow and heat transfer in internally finned tubes and ali," J. Heat Transfer, vol. 101, pp. 29-37, 1979.

[2] J. Y. Yang, M. C. Wu, and W. J. Chang, "Numerical and experimental studies of three-dimensional plate-fin and turbulent exchangers," Int. J. Heat Mass Transfer, vol. 39, pp. 3057-3066, 1996.

[3] I. Alam and P. S. Ghoshdastidar, "A study of heat transfer effectiveness of circular tubes with internal longitudinal fins having tapered lateral profiles," Int. J. Heat Mass Transfer, vol. 45, no. 6, pp. 1371-1376, 2002.

[4] Z. F. Dong and M. A. Ebadian, "A numerical analysis of thermally developing flow in elliptical duct with internal fins," Int. J. Heat Fluid Flow, vol. 12, no. 2, pp. 166-172, 1991.

[5] A. M. Huq and M. M. Rahman, "Experimental measurements of heat transfer in an internally finned tube," Int. Comm. Heat Mass Transfer. vol. 25 , no. 5 , pp. 619-630, 1998.

[6] T. Boufendi and M. Afrid, "Three-dimensional conjugate conduction-mixed convection with variable fluid properties in a heated horizontal pipe," Revue des Energies Renouvelables, vol. 8, pp. 1-18, 2005.

[7] S. Touahri and T. Boufendi, "Numerical study of the conjugate heat transfer in a horizontal pipe heated by Joulean effect," Thermal Science, vol. 16, no. 1, pp. 53-67, 2012.

[8] W. M. Yan and P. J. Sheen, "Heat transfer and friction characteristics of fin and tube heat exchangers," Int. Journal of Heat and Mass Transfer, vol. 43, pp. 1651-1659, 2000.
[9] B. Yu, J. H. Nie, Q. Wang, and W. Tao, "Experimental study on the pressure drop and heat transfer characteristics of tubes with internal wave-like longitudinal fins," Heat and Mass Transfer, vol. 35, pp. 65-73, 1999.

[10] C. C. Wang, W. L Fu, and C. T. Chang "Heat transfer and friction characteristics of typical wavy fin and tube heat exchanger," Exp. Thermal Fluid Science, vol. 14, pp. 174-186, 1997.

[11] S. W. Churchill and H. S. Chu, "Correlating equation for laminar and turbulent free convection from a horizontal cylinder," Int. J. Heat Mas. Transfer, vol. 18, pp. 1049-1053, 1975.

[12] S. V. Patankar, Numerical Heat Transfer and Fluid Flow, New York: McGraw-Hill, 1980.

[13] M. Ouzzane and N. Galanis, "Effects of parietal conduction and heat flux repartition on mixed convection near the entrance of an inclined duct (in French)," Int. J. Thermal Sciences, vol. 38, pp. 622-633, 1999.

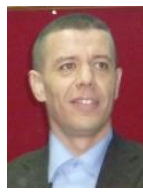

S. Touahri was born in 1977 in Guelma, Algeria. He received his DES (bachelor degree) in energy physics in 1998 from the Mentouri Brothers University of Constantine, Algeria, and the magister $(\mathrm{MSc})$ and doctorate $(\mathrm{PhD})$ in the same specialty and university in 2002 and 2012 respectively. $\mathrm{He}$ is currently an associate professor in energy physics and his research interest is the computational heat transfer and fluid dynamic, in particular the internal flows with conjugate heat transfer, and the renewable energy (especially solar).

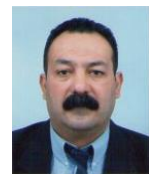

T. Boufendi was born in 1958 in Constantine, Algeria. He received the DES, magister and doctorate degrees from the Mentouri Brothers University of Constantine, in 1981, 1985 and 2005 respectively, all in energy physics. Since Dec. 2011, he has been a professor in the same university and his research interest include in the field of the computational heat transfer and fluid dynamic, in particular the internal flows with conjugate heat transfer, and the renewable energy (especially solar). 